\title{
IMPACTO DO USO DE DROGAS NOS RELACIONAMENTOS FAMILIARES DE DEPENDENTES QUÍMICOS
}

Miriam Aparecida Nimtz¹, Anna Maria Fornalski Tavares², Mariluci Alves Maftum¹, Aline Cristina Zerwes Ferreira³, Letícia de Oliveira Borba ${ }^{4}$, Fernanda Carolina Capistrano ${ }^{3}$

'Enfermeira. Doutora em Enfermagem. Universidade Federal do Paraná. Curitiba-PR-Brasil.

${ }^{2}$ Enfermeira. Curitiba-PR-Brasil.

${ }^{3}$ Enfermeira. Mestranda em Enfermagem. Universidade Federal do Paraná. Curitiba-PR-Brasil.

${ }^{4}$ Enfermeira. Doutoranda em Enfermagem. Universidade Federal do Paraná. Curitiba-PR-Brasil.

RESUMO: O objetivo desse trabalho foi identificar o impacto do uso de drogas nos relacionamentos familiares de dependentes químicos. Trata-se de pesquisa qualitativa exploratória, desenvolvida em uma unidade de reabilitação para dependentes químicos no estado do Paraná. Foram respeitados os princípios éticos de acordo com a Resolução 196/96. Os dados foram coletados por meio de entrevista semiestruturada, no período de agosto a setembro de 2012, com 20 dependentes químicos. Os dados foram tratados com a análise categorial temática. As categorias analisadas foram: Influência das relações familiares no uso de drogas e Quebra de vínculos familiares. Conclui-se que a dependência química é uma doença que afeta os relacionamentos, prejudicando a qualidade de vida das pessoas dependentes e de seus familiares. Assim, ressalta-se a importância de profissionais de saúde capacitados, visando atenção a esta clientela.

DESCRITORES: Relações familiares; Transtornos relacionados ao uso de substâncias; Saúde mental; Enfermagem.

\section{THE IMPACT OF DRUG USE ON THE FAMILY RELATIONSHIPS OF DRUG ADDICTS}

\begin{abstract}
This work aims to identify the impact of drug use on the family relationships of drug addicts. It is exploratory qualitative research, undertaken in a rehabilitation unit for drug addicts in the Brazilian state of Paraná. Ethical principles - in accordance with Resolution 196/96 - were respected. The data were collected through semistructured interviews, held in the period August September 2012 with 20 drug addicts. The data were treated using thematic categorical analysis. The categories analyzed were: Influence of family relationships on drug use and Breaking family bonds. It is concluded that drug addiction is an illness which affects the relationships, impairing the quality of life of the addicts and their family members. Thus, the importance of trained health professionals is emphasized, with a view to attention for this clientele.
\end{abstract}

DESCRIPTORS: Family relationships; Disturbances related to substance use; Mental health; Nursing.

\section{IMPACTO DEL USO DE DROGAS EN LAS RELACIONES FAMILIARES DE DEPENDIENTES QUÍMICOS}

RESUMEN: El objetivo de ese trabajo fue identificar el impacto del uso de drogas en las relaciones familiares de dependientes químicos. Es una investigación cualitativa exploratoria, desarrollada en una unidad de rehabilitación para dependientes químicos en el estado de Paraná. Fueron respetados los principios éticos de acuerdo con la Resolución 196/96. Los datos fueron obtenidos por medio de entrevista semiestructurada, en el periodo de agosto a septiembre de 2012, con 20 dependientes químicos y trabajados por medio del análisis categoríal temático. Las categorías analizadas fueron: Influencia de las relaciones familiares en el uso de drogas y Rompimiento de vínculos familiares. Se concluye que la dependencia química es una enfermedad que afecta los relacionamentos, perjudicando la cualidad de vida de las personas dependientes y de sus familiares. Así, se destaca la importancia de profesionales de salud habilitados, visando la atención a esa clientela. DESCRIPTORES: Relaciones familiares; Trastornos referentres al uso de substancias; Salud mental; Enfermería. 


\section{INTRODUÇÃO}

A família faz parte do desenvolvimento do indivíduo, nesse processo pode tornar-se um fator de proteção às drogas, ao diminuir a exposição a eventos estressantes que o indivíduo possa ter que enfrentar ou um fator de risco quando favorecem a suscetibilidade a problemas emocionais e comportamentais $^{(1)}$.

Conforme dados epidemiológicos, publicado em 2011 pelo Report on Alcohol and Health, o consumo de bebidas alcoólicas aumentou no Brasil, entre 2001 e 2005, para mais de $30 \%$ nos homens e entre $10 \%$ e $20 \%$ nas mulheres, sabendo que foram os percentuais mais altos das Américas. Contudo, teve-se como consequência desse consumo cerca de $10 \%$ das mortes no Brasil. Isto é determinante e revela que o alcoolismo, assim como as outras drogas, constitui sério problema de saúde pública, sendo uma realidade a presença de tais consumidores nos serviços de saúde em busca das emergências provindas da própria dependência ou de suas complicações ${ }^{(2-3)}$.

Acrescido a esses dados epidemiológicos, o Relatório brasileiro sobre drogas de 2009 mostra que o uso de álcool, nas 108 maiores cidades do país, foi de $74,6 \%$, porcentagem inferior a de outros países (Chile com 86,5\% e EUA com $82,4 \%)$, sendo o menor uso de álcool na Região Norte (53,9\%) e o maior na Sudeste (80,4\%). Em todas as regiões observaram-se mais dependentes de álcool para o gênero masculino. Além disso, 12,3\% dos brasileiros são dipsomaníacos (compulsivos por ingerir bebida alcoólica) e 2,1\%, incluindo ambos os sexos e todas as faixas etárias, são dependentes de outras drogas ${ }^{(4)}$.

Um dos prejuízos causado pelo consumo do álcool é a desestruturação familiar, pois viver em um ambiente assombrado pelo álcool desencadeia a probabilidade de afetar outros descendentes de alcoolistas. A literatura aponta que para cada alcoolista cinco ou seis pessoas da família são afetadas e adoecem, além de criar e manter desavenças, falta de credibilidade e desconfianças ${ }^{(1,4)}$.

No que concerne ao tratamento do alcoolismo, o mesmo depende de uma série de fatores, dentre eles a necessidade de cada usuário e do recurso disponível, tais como: Centros de Atenção
Psicossocial álcool e outras drogas - CAPS ad, serviços da atenção básica, ambulatórios, hospitais e grupos de apoio da comunidade. A família, além de necessitar de acompanhamento, precisa estar presente na reabilitação psicossocial, melhorar os relacionamentos, proporcionar o resgate da autonomia de cada um da família e, principalmente, encorajar o usuário e seus familiares para mudança. Há diretrizes do Ministério da Saúde que propõem tais componentes, inclusive que os CAPS ad devem realizar atividades de cunho preventivo/educativo tanto com os usuários quanto com os familiares ${ }^{(4-5)}$.

O desajuste familiar na primeira infância, por exemplo, pode culminar no uso/abuso de drogas, posteriormente. A partir desse contexto, supõe-se que as condições do ambiente familiar podem propiciar a geração de estados emocionais altamente estressantes e favoráveis ao uso de drogas ${ }^{(6)}$.

Com relação ao uso do crack, caracteriza-se por um alto potencial aditivo devido à intensa fissura que, posteriormente, estimula o consumo repetitivo e exacerbado. A compulsão instiga o dependente de crack a buscar, ligeiramente, tal substância por meio de comportamento de risco, que geram impactos sociais e pessoais ${ }^{(4,7)}$.

Conforme relatório da Associação Brasileira de Psiquiatria (2011), a cocaína e o crack são consumidos por $0,3 \%$ da população mundial, sendo que a maior parte dos usuários concentra-se nas Américas (70\%) e, na última década, o número de usuários aumentou. Assim, destaca-se que no Brasil, o consumo de crack dobrou e que a região sul foi a mais atingida, aumentando o uso de $0,5 \%$ para $1,1 \%$, seguida pela região sudeste, $\operatorname{com~} 0,8 \%{ }^{(8)}$.

Em relação ao tabagismo, a Organização Mundial da Saúde (OMS) aponta para a alta morbimortalidade, sendo aproximadamente cinco milhões de mortes ao ano e considerado a maior causa de morte evitável e de maior crescimento no mundo. Contudo, a maioria dos fumantes adquire o hábito de fumar e a dependência da nicotina, a priori, na adolescência, mediante influência do círculo de convívio social, o que pode comprometer tal dependência, por conseguinte, a vida adulta. Numa análise mais abrangente sobre os esforços no controle do tabagismo, a OMS alerta para que se não houver um esforço para diminuir o número de fumantes, o tabagismo poderá matar um bilhão de pessoas no século 21, nos países em desenvolvimento. 
Essa previsão significa 10 vezes mais mortes do que se previa no século passado( ${ }^{(9)}$. A realidade mostra que há grandes dificuldades com relação ao controle do consumo precoce de drogas no país por muitos motivos, dentre eles a relevante extensão geográfica, o que estimula a entrada de drogas no País. A divulgação em massa de substâncias psicoativas culmina em estímulo ao consumo das mesmas ${ }^{(10)}$.

Existem circunstâncias que mais chamam a atenção do indivíduo para usar a droga e que teriam ressonância e relação com: locais propícios; estar acompanhado por alguém que usa drogas, o que no meio familiar e na rede de amigos chamamos comumente de "más companhias"; ir a festas com os amigos; observar alguém usando ou desfrutando de drogas; sentir-se muito e ligeiramente ansioso, estressado ou frustrado porque "tudo está dando errado em sua vida"(11).

Acrescenta-se que fatores sociodemográficos, tais como: idade, sexo e aspectos psicossociais, como influência dos amigos e relações interpessoais dentro da família são importantes ao considerar o uso e abuso de drogas por jovens e adultos, o que justifica a importância desse estudo.

A partir do exposto, considera-se relevante a questão do uso de substâncias psicoativas e seu impacto nos relacionamentos familiares do dependente químico. Desse modo, o objetivo dessa pesquisa foi identificar o impacto, segundo dependentes químicos, do uso de drogas nos seus relacionamentos familiares.

\section{METODOLOGIA}

Pesquisa qualitativa exploratória, realizada no período de agosto a setembro de 2012, em uma unidade de reabilitação para dependentes químicos do sexo masculino e maiores de 18 anos, do estado do Paraná.

Os critérios de inclusão dos participantes na pesquisa foram: ter idade igual ou superior a 18 anos, estar em tratamento na unidade de reabilitação e não estar em período de desintoxicação.

A população total de pacientes em tratamento, durante a coleta de dados, constituía-se de 30 dependentes químicos e 20 fizeram parte desta pesquisa, definido pela saturação teórica dos dados.

Os dados foram coletados mediante entrevista semiestruturada e gravada, com a questão aberta:
"Fale-me sobre aspectos que você considera como consequência do uso de drogas em sua relação familiar".

A análise das informações obtidas das entrevistas ocorreu com o uso da técnica de análise categorial temática, que se organiza, operacionalmente, em três fases: pré-análise, exploração do material e tratamento dos resultados obtidos e interpretação $\mathrm{O}^{(12)}$. Dessa forma, as entrevistas foram transcritas na íntegra; posteriormente os dados foram sistematizados de modo a manter o conteúdo básico do pensamento e o significado da mensagem apresentada pelo dependente químico.

Os participantes da pesquisa foram descritos pela letra " $\mathrm{P}$ ", acrescido de um numeral sem correlação com a ordem das entrevistas, a fim de preservar o anonimato das informações. Este estudo faz parte do projeto "Reinternamento de dependentes químicos em uma Unidade de Reabilitação: causas e motivações da recaída", aprovado pelo Comitê de Ética em Pesquisa do Setor de Ciências da Saúde da Universidade Federal do Paraná (UFPR), sob o registro CEP/SD: 904.029.10.03; CAAE 0825.0000091.10.

\section{RESULTADOS}

A idade dos 20 participantes variou entre 21 e 66 anos, sendo 12 entre 20 a 40 anos e 17 participantes começaram a usar drogas entre 10 e 19 anos. A droga lícita mais comumente usada foi o álcool (20), seguida do tabaco (18); e a ilícita crack (11) e cocaína (oito). Destaca-se que todos os participantes faziam uso de mais de uma substância. Esses resultados mostram que os participantes são pessoas de idade adulta, tendo por consequência desajustes nas atividades laborais e de suas funções sociais e familiares.

A partir da análise de conteúdo encontrou-se como duas categorias para análise: Influência das relações familiares no uso de drogas e Quebra de vínculos familiares.

\section{Categoria 1 - Influência das relações familiares no uso de drogas}

Os depoimentos retratam que o comportamento familiar exerce influência sobre seus descendentes, uma vez que o histórico familiar pode contribuir para o surgimento do uso de drogas, especialmente se no convívio familiar 
existirem situações que exponham e afetem a saúde mental do indivíduo. Com este teor há os recortes a seguir:

Tenho uma tia aqui no norte, que bebe pinga de garrafão, toda tarde e ela gosta quando estou lá, porque tem um companheiro. (P.5)

Para falar a verdade, só tenho um irmão que não bebe, o restante bebe cachaça. (P.7)

Eu estava na casa do meu pai e ele fez duas caipirinhas. Ele costuma beber sempre comigo.(P.8)

Mas a culpa disso tudo era dos meus pais e do meu avô, que apoiavam a bebida desde pequeno. (P.11)

\section{Categoria 2 - Quebra de vínculos familiares}

As consequências do consumo de drogas repercutem em perda de confiança e quebra de vínculos familiares, sendo a separação conjugal a mais citada pelos participantes:

Eu não conseguia ter uma relação normal com a minha mulher por causa das drogas, o que resultou na separação. (P.1)

As drogas me afastaram da minha família, porque eu fiz algumas coisas que não devia onde eu morava e tive que me mudar. [...] A relação com a família ficou complicada também, eles perderam a confiança em mim. (P.3)

Com a minha ex-mulher sempre havia muitas discussões e brigas. [...] A separação estava relacionada às drogas. (P.13)

A separação com a minha mulher ocorreu porque comecei a beber bastante e pela condição financeira. (P.20)

\section{DISCUSSÃO}

Na síndrome da dependência, embora seja um evento multicausal, o comportamento familiar influencia, sobremaneira, seus descendentes, tanto que o histórico familiar pode contribuir para o mesmo transtorno, por uma cultura de aprendizado. Assim, esse ciclo torna-se constante, na medida em que tais pacientes já são pais e, consequentemente, já exercem tal influência sobre seus filhos ${ }^{(10,13-14)}$.

Enuncia-se que os cuidados nos primeiros anos de vida são de extrema importância para o indivíduo no que concerne a sua saúde mental. Assim, se no ambiente familiar existe situações que expõem e afetam a saúde mental do indivíduo, tais como abuso emocional e negligência física e emocional, indica o desenvolvimento de comportamentos tão adversos quanto àqueles já presenciados outrora, como: consumo de álcool, abuso de substâncias, tentativa de suicídio, e comportamento sexual de risco ${ }^{(15-16)}$.

Especificamente, por meio dos relatos, identificaram-se frequentes brigas nas relações maritais. Vários estudos ${ }^{(1,4-5,10,14)}$ atestam que as pessoas que crescem nesse ambiente adverso presenciam diversas contradições nas pessoas adictas, o que pode culminar na falta de definições claras das regras do sistema familiar. Deste modo, a droga serve como uma forma da pessoa adicta diferenciar-se de sua família de origem ou de chamar a atenção da mesma, devido ao empobrecimento das regras e dos limites.

Em relação às substâncias ilícitas verificadas no estudo, o crack superou as demais substâncias, situação que pode ser explicada ao levarmos em consideração o baixo custo da droga e o intenso prazer que ela proporciona. Corrobora com esse achado, o relatório da ONU de 2012, que apontou aumento no consumo de cocaína/crack, no Brasil, mas enfatizou também que a falta de dados novos impede um melhor entendimento do impacto nas estimativas regionais ${ }^{(17)}$.

Fato é que conviver com um dependente químico não é fácil, pois são frequentes as desavenças familiares e, consequentemente, separações conjugais, haja vista que o usuário de droga, mediante efeito dela, pensa de forma egoísta e está voltado ao consumo da substância pela qual está dependente; conflitos entre dependentes químicos e familiares são constantes, o que culmina, muitas vezes, em divórcios, restringindo inclusive o contato com os filhos ${ }^{(13)}$.

O impacto causado pelo uso de drogas nos relacionamentos familiares é variável e depende das relações que a mesma tem com seus 
membros usuários de drogas, suas características externas e internas, o momento do ciclo vital em que vive, a história intergeracional e o contexto sociocultural em que essa família está inserida ${ }^{(18)}$.

Indubitavelmente, questões envolvendo a estrutura da dinâmica familiar podem ser um dos fatores que contribuem para o uso de drogas, tornando-se importante frisar que a família está implicada no desenvolvimento saudável ou não de seus membros, pois é o elo entre as diversas esferas da sociedade ${ }^{(19)}$.

A interação dos membros da família em seus respectivos papéis determina a qualidade da estabilidade das relações familiares. A conquista da estabilidade é influenciada pela capacidade de seus membros controlarem seus conflitos internos e relacionais. Assim, a família desempenha um papel crucial no processo de desenvolvimento dos que a constituem, é ela quem apresenta e estabelece as ligações emocionais, comunicacionais e afetivas entre seus membros e nas relações sociais, sendo um dos principais fatores de risco ou proteção no envolvimento dos mesmos com drogas ${ }^{(19)}$.

O consumo de drogas pode ser discutido a partir do papel que a família terá no tratamento, sendo que muitas vezes as famílias estão tão doentes quanto os seus membros, dependentes químicos. Deste modo, a mesma necessita transformar a visão que tem sobre si, de vítima a coparticipante, de culpada a corresponsável, de impotente a competente ${ }^{(20)}$.

O comportamento aditivo causa danos sociais inenarráveis, haja vista que os usuários acabam se afastando dos seus trabalhos não tendo a mesma produtividade, e necessitam de serviços de saúde de alta complexidade. Entretanto, tais serviços devem adequar-se a alta demanda atual de dependentes químicos, visando o melhor tratamento ${ }^{(14)}$.

Dessa forma, as drogas geram relevante impacto na vida dos usuários, levando-os às perdas físicas e psíquicas. Elas são responsáveis pela perda de emprego, de bens materiais, de rompimento dos vínculos familiares e, também, malefícios à saúde. Assim sendo, manter relacionamentos conjugais e parentais é muito difícil, na medida em que o usuário tem a tendência de substituir o relacionamento em sociedade pelo relacionamento com a droga ${ }^{(11)}$.

\section{CONCLUSÃO}

A relevância em entender a dinâmica familiar nos fatores orgânicos, psicológicos, socioculturais, espirituais, incluindo aqui a drogadição, está relacionada ao desenvolvimento saudável ou não de seus membros, pois ela é o sistema mais próximo do dependente químico entre as inúmeras esferas da sociedade.

Faz-se importante que os profissionais que assistem aos usuários, em especial os Enfermeiros, façam a diferença, prestando assistência integral e humanizada, bem como é fundamental a atuação dos demais profissionais da saúde na terapia familiar, que pode ser incluída no tratamento da dependência química, visando restabelecer o relacionamento do paciente com sua família, ajudando-os a lidarem com os enfrentamentos que a dependência química causa.

Atualmente existe uma rede de atenção à saúde que oferece tratamento ao dependente químico, visando à integração social do usuário, especialmente voltada ao acompanhamento sociofamiliar eà inclusão de crianças e adolescentes. Todavia, a mesma está se adequando a realidade da demanda dessa população, principalmente no que se refere à quantidade dos recursos oferecidos e na abordagem proporcionada ao usuário dependente e seus familiares.

Acrescido aos fatos expostos há necessidade de mais pesquisas, procurando compreender o papel da família que pode apresentar tanto fatores de risco quanto protetores, assim como a maior compreensão não apenas de quais são estes fatores, mas também como reduzir os fatores de risco e priorizar os fatores de proteção neste ambiente.

\section{REFERÊNCIAS}

1. Martins M, Santos MA, Pillon SC. Percepções de famílias de baixa renda sobre o uso de drogas por um de seus membros. Rev. Latino-Am. Enfermagem. [Internet] 2008;16(2) [acesso em 10 ago 2013]. Disponível: http:// dx.doi.org/10.1590/S0104-11692008000200019

2. Silva LHP, Borba LO, Paes MR, Guimarães AN, Mantovani MF, Maftum MA. Perfil dos dependentes químicos atendidos em uma unidade de reabilitação de um hospital psiquiátrico. Esc. Anna Nery. [Internet] 2010;14(3) [acesso em 11 ago 2013]. Disponível: http:// dx.doi.org/10.1590/S1414-81452010000300021 
3. Vargas D, Bittencourt MN, Rocha FM, Oliveira MAF. Representação social de enfermeiros de centros de atenção psicossocial em álcool e drogas (CAPS AD) sobre o dependente químico. Esc. Anna Nery. [Internet] 2013;17(2) [acesso em 18 ago 2013]. Disponível: http:// dx.doi.org/10.1590/S1414-81452013000200006

4. Brasil. Presidência da República. Secretaria Nacional de Políticas sobre Drogas. Relatório brasileiro sobre drogas. Secretaria Nacional de Políticas sobre Drogas, IME USP [Internet]. Brasília; 2009 [acesso em 15 ago 2013]. Disponível: http://www.obid. senad.gov.br/portais/OBID/biblioteca/documentos/ Relatorios/328379.pdf

5. Silva AC, Bortolotto CM, Mazzali DB, Martins DS, Martini JS, Marques LM, et al. Situações de recaída e família de origem: um estudo sobre a percepção de dependentes químicos. In: $4^{\mathrm{a}}$ Jornada de Pesquisa em Psicologia: Desafios atuais nas práticas da psicologia; 2011 Nov. p. 25-26; Santa Cruz do Sul - SC, Brasil. Santa Cruz do Sul: UNISC; 2011.

6. Terroso LB, Argimon IIL. Drogadição e adolescência: uma revisão. Psicologia. pt. [Internet] 2013;1(8) [acesso em 18 set 2013]. Disponível: www.psicologia.pt/ artigos/textos/A0709.pdf

7. Ferreira ACZ, Capistrano FC, Maftum MA, Kalinke LP, Kirchhof ALC. Caracterização de internações em uma unidade de reabilitação. Cogitare enferm. 2012;17(3):444-51.

8. Matos JC, Mello JM, Colombo JVP, Melo SR. Efeitos neurológicos da exposição pré-natal à cocaína/crack. Arq. Mudi. [Internet] 2011;15(1/2/3) [acesso em 19 set 2013]. Disponível: http://periodicos.uem.br/ojs/index. php/ArqMudi/article/view/21067

9. World Health Organization (WHO). Report on the global tobacco epidemic: Enforcing bans on tobacco advertising, promotion and sponsorship. [Internet] Geneva; 2013. [acesso em 15 ago 2013]. Disponível: http://apps.who.int/iris/ bitstream/10665/85380/1/9789241505871_ eng.pdf

10. Capistrano FC, Ferreira ACZ, Silva TL, Kalinke LP, Maftum MA. Perfil sociodemográfico e clínico de dependentes químicos em tratamento: análise de prontuários. Esc. Anna Nery. [Internet] 2013;17(2) [acesso em 15 ago 2013]. Disponível: http://dx.doi. org/10.1590/S1414-81452013000200005

11. Gabatz RIB, Johann M, Terra MG, Padoin SMM, Silva AA, Brum JL. Percepção do usuário sobre a droga em sua vida. Esc. Anna Nery. [Internet] 2013;17(3) [acesso em 10 out 2013]. Disponível: http://dx.doi.org/10.1590/ S1414-81452013000300016

12. Bardin L. Análise de Conteúdo. $3^{a}$ ed. Lisboa: Edições 70; 2000 .
13. Oliveira VC. Perfil sociodemográfico, clínico e familiar de dependentes químicos em tratamento em um Centro de Atenção Psicossocial - Álcool e Outras Drogas da região metropolitana de Curitiba [dissertação]. Curitiba (PR): Universidade Federal do Paraná; 2011. p.47-48.

14. Gabatz RIB, Schmidt AL, Terra MG, Padoin SMM, Silva AA, Lacchini AJB. Percepção dos usuários de crack em relação ao uso e tratamento. Rev. Gaúcha Enferm. [Internet] 2013;34(1) [acesso em 16 ago 2013]. Disponível: http://dx.doi.org/10.1590/S198314472013000100018

15. Souza J, Carvalho AMP. Filhos adultos de pais alcoolistas e seu relacionamento na família de origem. Saúde \& Transformação Social. [Internet] 2012;3(2) [acesso em 15 ago 2013]. Disponível: http://www. incubadora.ufsc.br/index.php/saudeetransformacao/ article/view/1094

16. Scaduto AA, Barbieri V. O discurso sobre a adesão de adolescentes ao tratamento da dependência química em uma instituição de saúde pública. Cienc. saude colet. [Internet] 2009;14(2) [acesso em 10 ago 2013]. Disponível: http://dx.doi.org/10.1590/S141381232009000200029

17. World Health Organization (WHO). United Nations Office on Drugs and Crime. World drug report. New York: United Nations Office on Drugs and Crime; 2012.

18. Arald JC, Njaine K, Oliveira MC. Família e escola: uma parceria possível na prevenção de uso de drogas entre adolescentes. In: Osório LC, Valle ME. Manual de Terapia Familiar. Volume II. Porto Alegre: Artmed; 2010. p. 59-70.

19. Zacharias DG, Garcia EL, Petry ELS, Bringmann G, Skolaude LN. Familiares de usuários do crack: da descoberta aos motivos para o uso da droga. In: $4^{\text {a }}$ Jornada de Pesquisa em Psicologia: Desafios atuais nas práticas da psicologia; 2011 Nov. p. 16-29; Santa Cruz do Sul - SC. Brasil. Santa Cruz do Sul: UNISC; 2011.

20. Guimarães FL, Costa LF, Pessina LM, Sudbrack MFO. Famílias, adolescência e drogadição. In: Osório LC, Valle ME. Manual de terapia familiar. Porto Alegre: Artmed; 2009. p. 350-65. 